\title{
The use of hydroxyethyl starch130/0.4 in major burns: a retrospective study
}

\author{
Guilabert P., Usúa G., Abarca L., Martín N., Salazar K., Sharp A.
}

Background and Goal of Study: Major burns require a huge resuscitation with more fluids than any other traumatic patient. The use of Hydroxyethyl Starches (HES) in these patients was a normal clinical practice until 2013, when the PRAC (Pharmacovigilance Risk Assessment Committee) issued an alert about the use of HES in septic, critical care patients and burns(1), but the studies in which the recommendations were based did not include major burn patients(2). After reviewing the evidence in burns there were no studies showing bad outcomes with the use of HES in burns(2,3). We carried on a retrospective study in our burn unit to determinate if the use of HES 130/0.4 in Burns is related to an increased mortality.

Material and Methods: We carried on a retrospective cohort study on mortality associated to volume replacement with HEA 130/0.4 during the period 2012-2015 in patients older than 18 years who were admitted in Vall d'Hebron University Hospital Burn Unit with burns in more than $20 \%$ of the Body Surface Area (BSA). The main objective was to determine whether the use of HEA 130/0.4 was associated with increased mortality in the large burned patient at 28 days (M28). The secondary objective was to see if the use of HEA $130 / 0.4$ was also related to an increase in mortality at 90 days (M90).

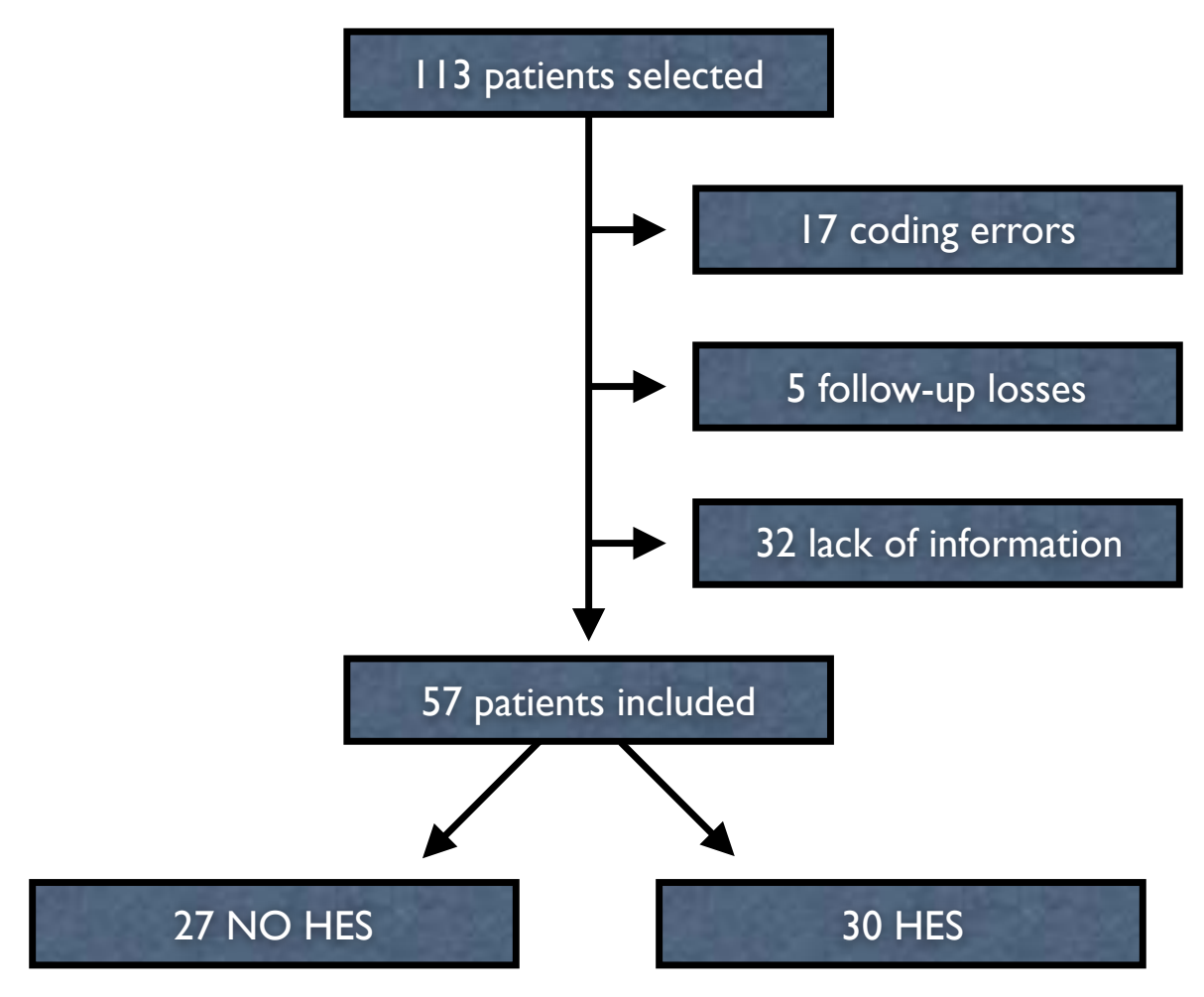

\begin{tabular}{|c|c|c|}
\hline AGE & Mean & Standard deviation \\
\hline NO HES & 45.48 & 16.33 \\
\hline HES & 52.87 & 21.19 \\
\hline
\end{tabular}

\begin{tabular}{|c|c|c|}
\hline BSA & Mean & Standard deviation \\
\hline NO HES & 41.26 & 19.34 \\
\hline HES & 40.80 & 22.62 \\
\hline
\end{tabular}

NO HES

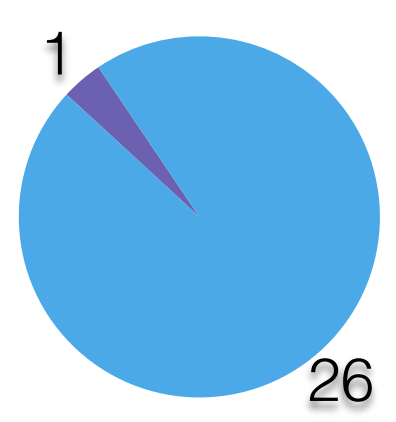

HES

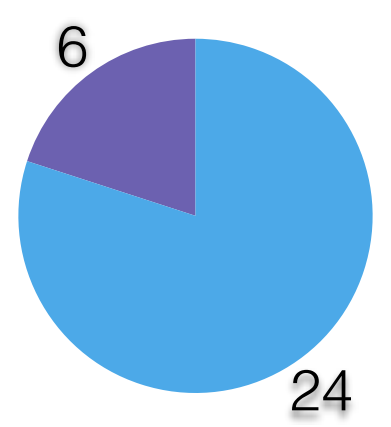

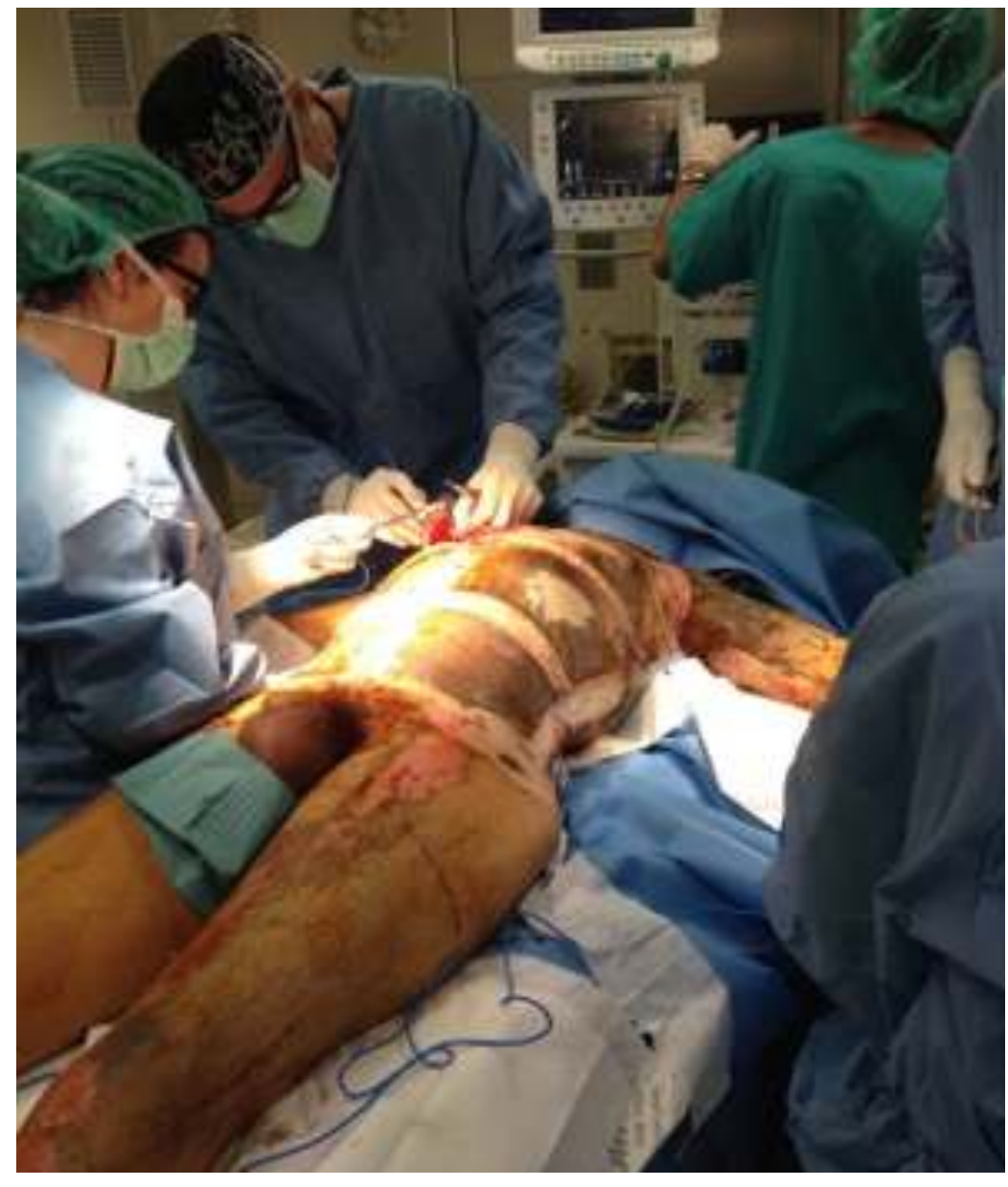

Results and Discussion: Of the 113 patients initially selected through coding in the database, 17 were excluded due to coding errors, 5 were follow-up losses due to relocation to other centers in the study period 32 were excluded due to lack of information, and 2 because of a limitation of therapeutic effort from admission. Of the 57 remaining, 30 received HES and 27 did not receive it. In the HES group the mean score of the ABSI (Acute Burn Severity Injury) scale was 9.40 whereas in the No-HES group it was 9.07 (non statistically significant differences). Mortality at 28 days was $10 \%$ in the HES group and $29.6 \%$ in the No HES and at 90 days $32.1 \%$ in the HES and $32.0 \%$ in the HES, in neither case the results were statistically significant.

\begin{tabular}{|c|c|c|}
\hline ABSI & Mean & Standard deviation \\
\hline NO HES & 9.07 & 2.81 \\
\hline HES & 9.40 & 2.47 \\
\hline
\end{tabular}

Wilcoxon test showed no significant differences between groups in ABSI score.

\begin{tabular}{|c|c|c|}
\hline MORTALITY & M28 & M90 \\
\hline NO HES & $29,60 \%$ & $32,00 \%$ \\
\hline HES & $10 \%$ & $32,10 \%$ \\
\hline$p$ & 0,061 & 0,991 \\
\hline
\end{tabular}

Results for Chi-square test. Fisher 2 tails test correction were 0.093 for M28 and 1.00 for M90

Conclusions: There were no significant differences in mortality at day 28 and 90 with the use of HES 130/0.4 in major burns. More prospective randomized studies about this issue are needed. 